\title{
Novel simulation technique for the prediction of complex oxidation behaviour in natural graphite flakes
}

\author{
Heinrich Badenhorst \\ SARChl Chair in Carbon Materials and Technology, Department of Chemical Engineering, University of Pretoria, \\ Lynnwood Road, Pretoria, 0002, South Africa \\ Tel: +27 12420 4173. Fax: +27 12420 2516. E-mail: carbon@up.ac.za
}

\begin{abstract}
The active surface area progression which takes place in natural graphite flakes during oxidation is difficult to model analytically. A probability based simulation was developed to represent the observed behaviour. The simulation is simple and capable of easily representing complex geometries such as randomized structures or curved contours which would be difficult and time consuming to model analytically. The probability used in the simulation is directly linked to the kinetic parameters.

These parameters and conversion behaviour were experimentally measured for a high purity, flake natural graphite sample. In addition, the sample was partially oxidized and extensively examined under a scanning electron microscope. Three characteristic microstructures were identified in the oxidized sample. These structures were qualitatively approximated by three simulated structures. Two of the three simulations were unable to effectively represent the initial region of the conversion function where it increases towards a maximum.

The complete behaviour was accurately represented by the simulation of a square flake with a few randomly positioned pits growing in the flake structure. The simulation illustrates that the initial increase is due to the growth of the pits within the larger flake structure. The pits were found to be caused by catalytic impurities. Thus by demonstrating this is a rate controlling factor the simulation enables the practical insight that the oxidation resistance can be significantly improved if these small impurities are removed.
\end{abstract}

Keywords: Probability simulation, Reaction interface, Natural graphite, Oxidation, Conversion function 


\section{Introduction}

Graphite is an important industrial material and is used in many high-temperature applications, ranging from structural and moderator components in nuclear reactors to electrodes for arc furnaces in the steel production industry. Its use is also increasing in electronic and industrial heat management, intumescent polymer additives, insulation panels and pressed graphite sheets for automotive and fluid sealing applications. Thus knowledge of the high-temperature oxidative behaviour of graphite is essential to understanding any degradation which may occur due to oxidation during normal operation. In the case of nuclear reactors, it is particularly important for ensuring safety and core stability during abnormal conditions such as air ingress into the reactor.

Natural graphite has an apparently simple, flat, flake-like structure, making it an ideal starting material for modelling. However, it has recently been shown [1] that these flakes can exhibit complex behaviour as conversion proceeds across the entire range. The isothermal conversion curve cannot be adequately represented by analytical models. The reason for this deficiency is related to random distributions of defects and impurities in the material which lead to the development of an intricate microstructure during oxidation. Thus an opportunity exists to develop a simulation technique to accurately represent this behaviour and the development of the flake microstructure across the entire conversion range. This enables a clear understanding of the mechanisms which govern the oxidative properties of the material.

The aim of this investigation was to develop a technique to easily simulate and predict the microstructural progression during the oxidation or gasification of complex, two dimensional shapes. The technique was first validated by comparing a simulated disc model to the analytical model for a disc. It was then used to simulate the conversion behaviour of natural graphite flakes during oxidation. The flakes were oxidized in pure oxygen at atmospheric pressure. The model geometries were based on three microstructures directly observed in the graphite. To assess the validity of these models, the simulated conversion functions were compared to the experimentally determined function.

Since this approach is linked to the real microstructural development occurring in the material it may be used to understand the changes that occur in other physical properties during operational degradation via oxidation. The simulation provides practical insights into the behaviour of the material thus enabling the identification of means to improve the properties.

\section{Theory}


Graphite is a solid reactant that reacts directly with gaseous oxygen to produce gaseous carbon dioxide or carbon monoxide, depending on conditions [2]. In general, graphite materials can have a very high purity and secondary reactions are limited with virtually no ash generation. Graphite has a well known crystal structure [3] as shown in Figure 1.

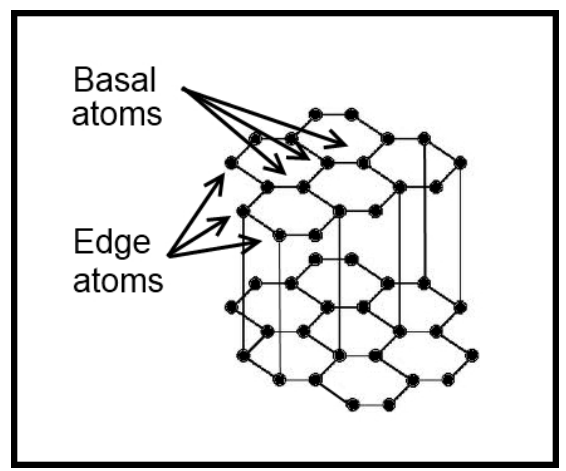

Figure 1: Ideal graphite structure

Natural graphite flakes are generally considered to be effectively single crystals $[4,5]$. When natural graphite flakes are examined under a scanning electron microscope (SEM), they are roughly square or disc shaped, with some irregularities along the edges, as can be seen from Figure 2.

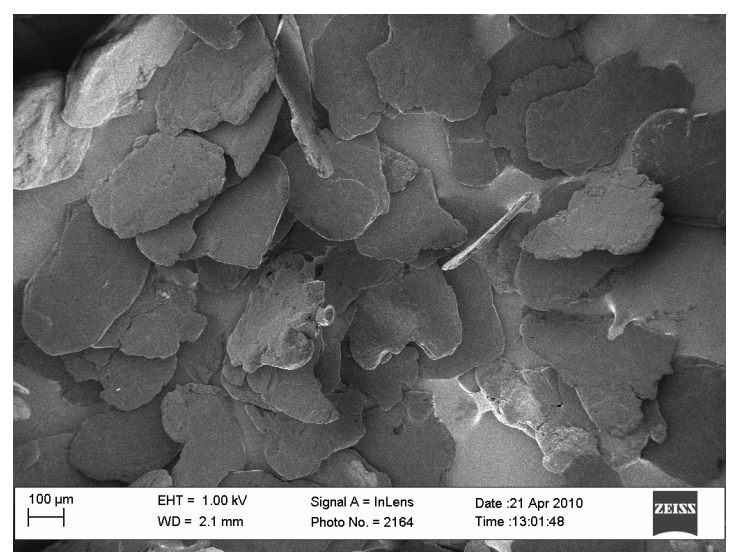

Figure 2: Graphite flake shapes

The flakes are solid with a very high aspect ratio. The structure of ideal graphite is layered, planes of hexagons forming a honeycomb structure, due to the $\mathrm{sp}^{2}$ hybridisation of the carbon atoms. Adjacent layers are weakly bonded to each other by van der Waals forces. When the real particles are examined more closely, as in Figure 3, the layered structure becomes clear and the edges can be seen to be fairly uniform and flat without porosity. This is consistent with expectations for highly crystalline natural graphite flakes [6]. 


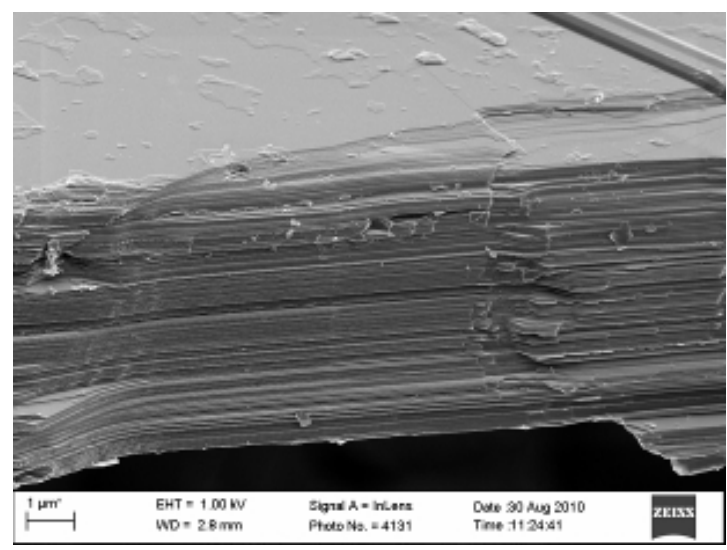

Figure 3: Graphite flake edge

Due to the strong, in-plane covalent bonding, the basal plane of graphite, i.e. the flat flake surface, is considered to be comparatively inert. Thus attack by oxygen is only possible at the edge atoms shown in Figure 1, except at extreme temperatures. This edge area is also known as the active surface area (ASA) and the reaction rate is controlled by this interface. The solid state kinetic approach is widely used to represent the reactions of solids with gases [7]. It has recently been shown [1] that a generalized expression for reaction interface controlled solid reactivity is given by:

$$
\frac{d \alpha}{d t}=\frac{A S A_{0}}{m_{0}} k^{A S A}\left(T, P^{o_{2}}\right) f^{A S A}(\alpha)
$$

Where $\alpha$ is the dimensionless degree of conversion and the conversion function $f^{A S A}(\alpha)$, is the normalized progression of the active surface area. Furthermore $k^{A S A}\left(T, P^{o_{2}}\right)$ is the reaction rate constant or turnover frequency based on the active surface area. It should be noted that since the current investigation was conducted in pure oxygen, the dependence on the gaseous reactant partial pressure reduces to unity. The dimensionless degree of conversion, $\alpha$, varies from zero to one as the reaction proceeds to full conversion and is defined as:

$$
\alpha=\frac{m_{0}-m}{m_{0}}
$$

Where $m$ and $m_{0}$ are the sample mass at any time $t$ and at the initial time $t_{0}$ respectively. For a small incremental time step, $\Delta t$, the change in mass may be approximated as:

$$
d m \approx \Delta t \frac{d m}{d t}
$$

Since $f^{A S A}(\alpha)=A S A / A S A_{0}$ and by taking the derivative of expression (2) it may be shown that: 


$$
d m \approx \Delta t \frac{d m}{d t}=-\Delta t m_{0} \frac{d \alpha}{d t}=-\Delta t k^{A S A}\left(T, P^{o_{2}}\right) A S A
$$

To represent the active surface based reaction behaviour of graphite consider an infinitesimal cube at the edge of an infinite flat sheet of thickness, $d$, shown in Figure 4.

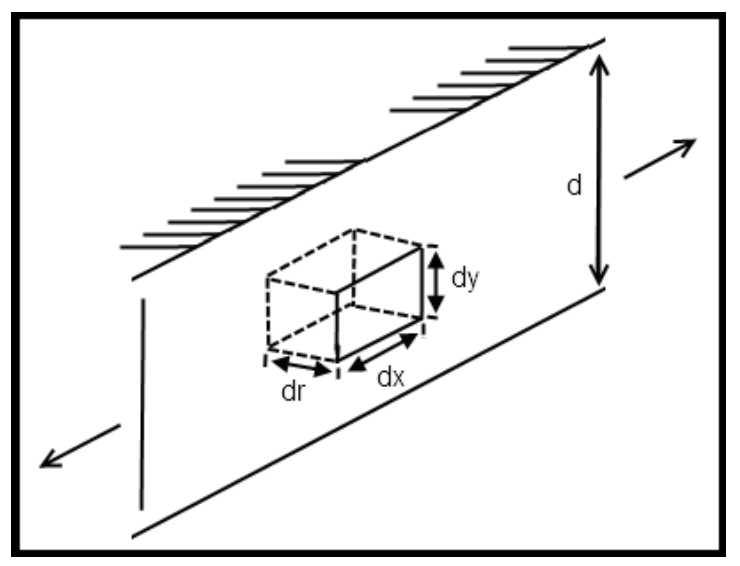

Figure 4: Infinitesimal cube at the edge of an infinite flat sheet

Based on this the mass loss during a time step may be alternatively formulated using the probability that an infinitesimal cube was reacted away, $P_{\text {cube, }}$ as:

$$
d m=-P_{\text {cube }} n_{\text {edge }} m_{\text {cube }}
$$

Where $n_{\text {edge }}$ is the number of cubes exposed at the edge and $m_{\text {cube }}$ is the mass of a single cube. It is clear that:

$$
\begin{aligned}
& m_{\text {cube }}=d r d x d y \rho_{G} \\
& A S A_{\text {cube }}=d x d y
\end{aligned}
$$

Where $\rho_{G}$, is the density of crystalline graphite $\left(2.26 \mathrm{~g} / \mathrm{cm}^{3}\right)$. The number of cubes exposed at the edge is simply the total active surface area $(A S A)$ divided by the active surface area of a single cube. Thus by equating expressions (4) and (5) and substituting in the relevant terms, one finds that:

$$
P_{\text {cube }}=k^{A S A}\left(T, P^{o_{2}}\right) \frac{\Delta t}{d r \rho_{G}}
$$

Thus the reaction probability may be directly calculated from the measured reaction rate constant and a suitable selection of the simulation parameters, $\Delta t$ and $d r$. Since the reaction probability must always be less than or equal to one, these two parameters are not independent. For cases where reaction is possible from multiple faces it is important to maintain a constant reaction rate per unit active surface area. All cubes have the same mass but multi-sided cubes have a linearly 
increasing active surface area. In order to maintain the same reaction rate the reaction probability must linearly increase. Hence the reaction probability for an $n$-sided cube is given by:

$$
P_{\text {cube }}^{n \text { sided }}=n P_{\text {cube }}
$$

However this places the maximum limit on the reaction probability at $P_{\text {cube }}=0.25$, if the linear reaction probability relationship is to be maintained up to and including four sided cubes. This probability relationship can now be used to simulate the conversion of natural graphite flakes. First the flake shape is subdivided into a grid of small sub-elements analogous to a block cellular automaton. For example the circular disc shown in figure 5.

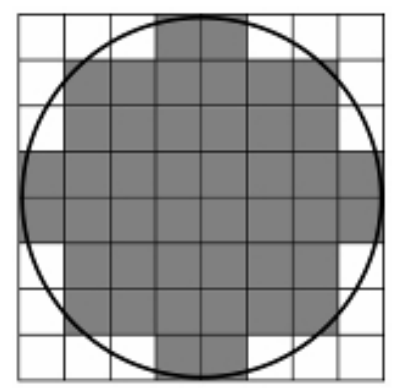

Figure 5: Grid approximation of a disc

For simplicity only a single layer is considered, this is not unreasonable bearing in mind that multiple layers of different, but similar sizes will simply lead to an averaging effect, much like a particle size distribution. During each time step a random number generator is used to spawn a number between zero and one for every sub-element with one or more exposed edges. If the number is less than the reaction probability the sub-element is deemed to have reacted and is removed from the simulation. This process is repeated until no elements are left.

The active surface area at any point is easily calculated by summing together all the exposed edges. By dividing this number by the number of exposed edges present initially, a scaled conversion function is obtained.

\section{Experimental}

Natural graphite flakes with a high purity of $>99.9 \%$ wt, designated RFL, were obtained from Graphit Kropfmühl AG (Germany). The sample was purified by the supplier using an acid wash and a high temperature soda ash burn. The sample was sieved to obtain a narrow particle size distribution of $200-250 \mu \mathrm{m}$. The sample was subjected to non-isothermal oxidation in pure oxygen at three different ramp rates: 3,4 and $5^{\circ} \mathrm{C} \cdot \mathrm{min}^{-1}$. All thermal oxidation was conducted in a TA Instruments SDT Q600 thermogravimetric analyzer (TGA). SEM images were obtained using an ultra-high 
resolution field-emission microscope (Zeiss Ultra Plus 55 FEGSEM) equipped with an in-lens detection system.

\section{Results}

The experimentally determined conversion behaviour is shown in Figure 6 .

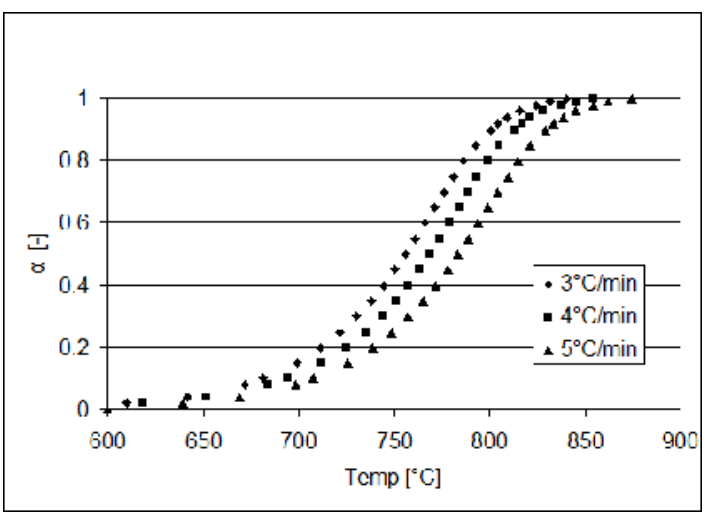

Figure 6: Observed conversion as function of temperature

The International Council for Thermal Analysis and Calorimetry (ICTAC) recommended [8] approach for analyzing solid state kinetic data was followed. The differential isoconversional method was used to estimate the activation energy. A simple Arrhenius expression was used for the temperature dependence of the reaction rate constant. The results are shown in Table 1.

Table 1. Kinetic results for RFL sample

\begin{tabular}{|c|c|c|c|c|}
\hline Sample & $\begin{array}{l}\text { Calculated } \\
\text { activation energy } \\
\left(\mathrm{kJ} . \mathrm{mol}^{-1}\right)\end{array}$ & $\begin{array}{l}\text { Std Dev } \\
\left(\mathrm{kJ} \cdot \mathrm{mol}^{-1}\right)\end{array}$ & $\begin{array}{l}\text { Std Dev } \\
(\%)\end{array}$ & $\begin{array}{l}\text { Pre-exponential } \\
\text { factor } \mathrm{k}_{0}^{\mathrm{ASA}} \\
\left(\mathrm{g} \cdot \mathrm{s}^{-1} \cdot \mathrm{m}^{-2} \mathrm{ASA}\right)\end{array}$ \\
\hline RFL & 157.7 & 4.2 & 2.7 & $2.4 \times 10^{6}$ \\
\hline
\end{tabular}

The reaction temperature was chosen as $750{ }^{\circ} \mathrm{C}$, which is well within the tested region. This was done to maintain the reaction probability below a value of 0.25 and preserve the consistency of the probability relationship. The experimentally obtained conversion function is shown in Figure 7. 


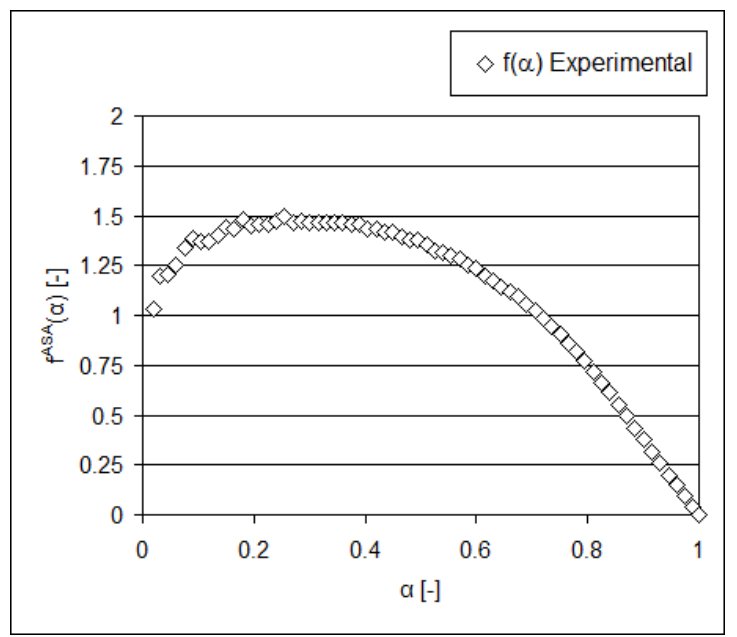

Figure 7: Experimental conversion function

\section{Modelling and simulation}

\subsection{Model validation}

The model kinetic parameters are set to the values obtained for the RFL graphite sample. As a starting point, a circular disc is simulated. The remaining model parameters are shown in Table 2 , chosen to be representative of the sample under consideration.

Table 2. Model parameters for circular disc

\begin{tabular}{lll}
\hline Parameter & Value & Units \\
\hline$D$ & 200 & $\mu \mathrm{m}$ \\
$d y=d$ & 20 & $\mu \mathrm{m}$ \\
$d x=d r$ & 2 & $\mu \mathrm{m}$ \\
$\Delta t$ & 5 & $\mathrm{~s}$ \\
\hline
\end{tabular}

Where $D$ is the particle diameter. Since the sub-element size is $2 \mu \mathrm{m}$ the starting grid size is 100 $x 100$ elements. It may be shown that the analytical model for a circular disc is given by the expression [1]:

$$
f^{A S A}(\alpha)=(1-\alpha)^{0.5}
$$

For comparative purposes this model is included in Figure 8. Figure $8 \mathrm{~A}$ through $\mathrm{C}$ demonstrates the effect of increasing the grid size to $1000 \times 1000$ (i.e. decreasing the element size to $0.2 \mu \mathrm{m}$ ). 

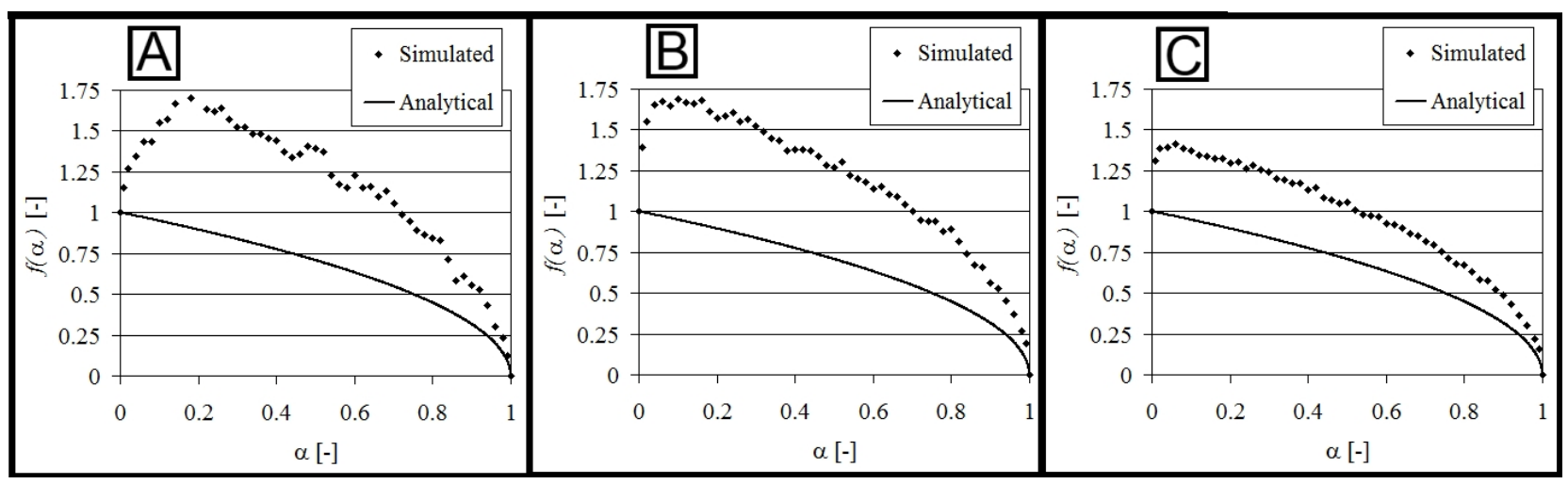

Figure 8: Simulated conversion function for different grids (A) 100x100 (B) 500x500 (C) $1000 \times 1000$.

Initially the approximation is fairly poor with a large amount of scatter due to the large subelement size. As the grid size is increased the simulation delivers an improved approximation. The final grid size provides an adequate representation of the expected analytical function. However, a discrepancy still exists at low conversion where the simulated conversion function shows an increase relative to the analytical model. This difference is made clear when the ratio of the two functions is plotted in Figure 9.

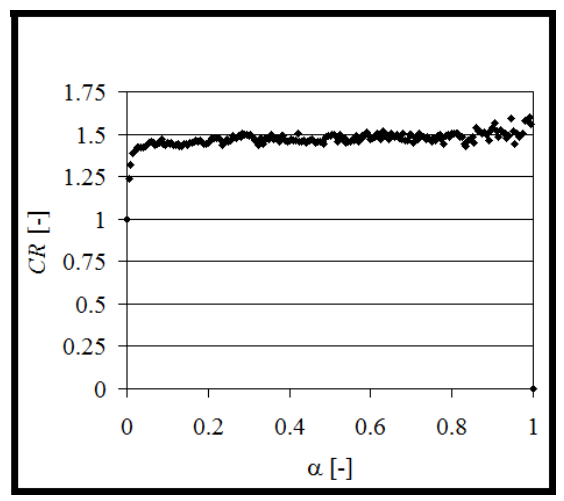

Figure 9: Conversion function ratio (CR)

The ratio starts off at a value of one and then rapidly increases to a value of around 1.47 and as conversion increases the ratio increases slightly. The reason for this ratio is the discrete nature of the simulation and the resulting edge roughness. The analytical model assumes a perfectly smooth surface, however since sub-elements are removed from the edge in a random, erratic fashion, this edge undergoes roughening until a pseudo steady state is reached. The value of this roughness and the speed with which steady state roughness is achieved, is only a function of the chosen grid size 
and the reaction probability. If the conversion function is divided by this constant value, the simulation gives an excellent approximation of the analytical disc model, as can be seen in Figure 10.

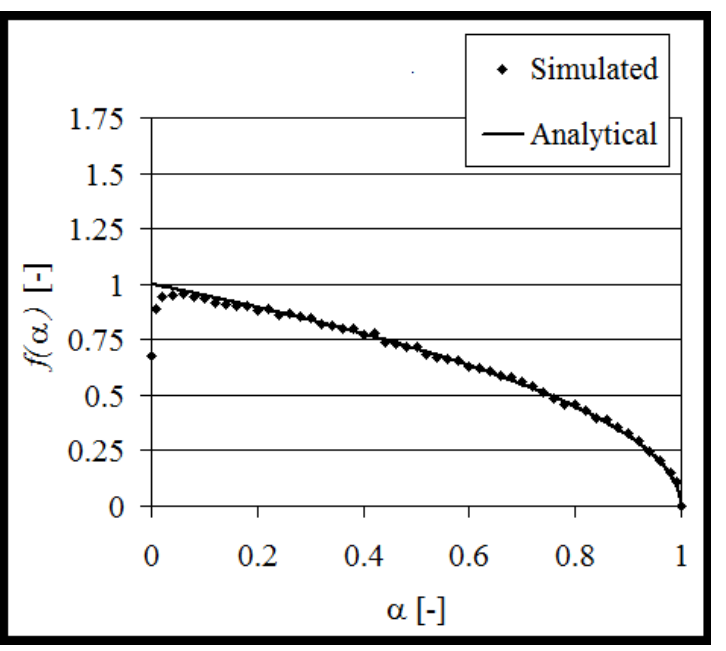

Figure 10: Scaled conversion function

Given these model parameters the time taken to achieve steady state roughening is very short and the only visible deviation from the analytical behaviour is at very low conversion. In a sense this simulation provides a more accurate representation of the true behaviour since the real sample will inevitably undergo the same edge roughening phenomenon. The small amount of drift as conversion increases is due to the increase of the roughening effect relative to the remaining particle size. However, this has a negligibly small impact on the overall behaviour as can be seen from Figure 10 where a constant ratio was used. This simple approach is capable of simulating complex edge features like those shown in Figure 11. In addition, it is capable of providing the active surface area progression of surfaces like randomly curved contours, which would be very challenging to represent analytically.

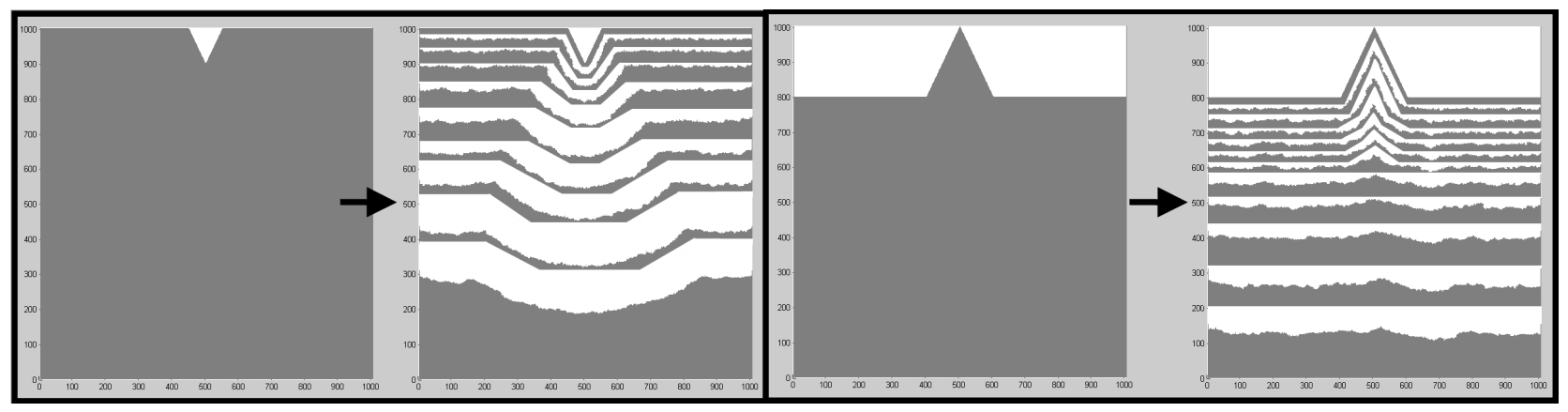

Figure 11: Complex edge recessions

\subsection{Observed graphite microstructures}


Visual inspection of the oxidized microstructure (at around 30\% burn-off) of the RFL graphite flakes in the SEM revealed several characteristic formations. Firstly, large fissures cutting across the flake macrostructure were observed as in Figure 12A. There is evidence that these are due to mineral inclusions in the flakes.

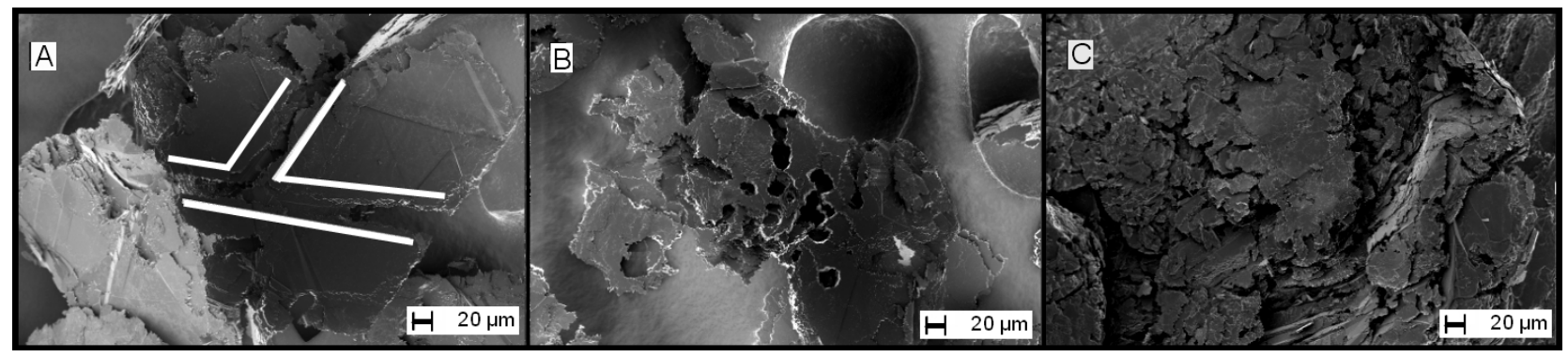

Figure 12: Oxidized RFL flakes with different microstructures.

In addition to the fissure structures, a variety of other microstructures were readily observable, such as the structure with circular pits shown in figure 11B. More erratic and complex microstructures were also discernable, seen in figure $11 \mathrm{C}$. These are presumably caused by defect-rich or deformed regions in the flake structure.

\subsection{Simulated microstructures}

Based on these observations, three simulated microstructures were formulated to describe the RFL structures as shown in Figure 13 to 15, designated SIM 1 through 3 respectively.

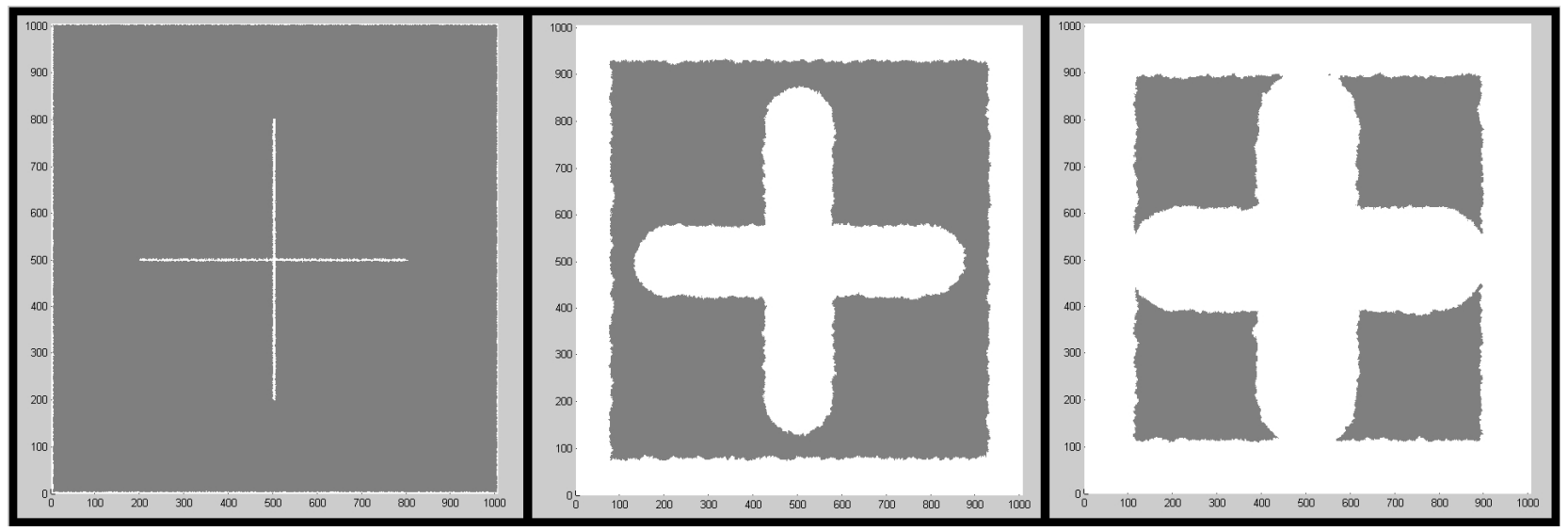

Figure 13: Reconstruction of flake structure with fissures (SIM 1). 


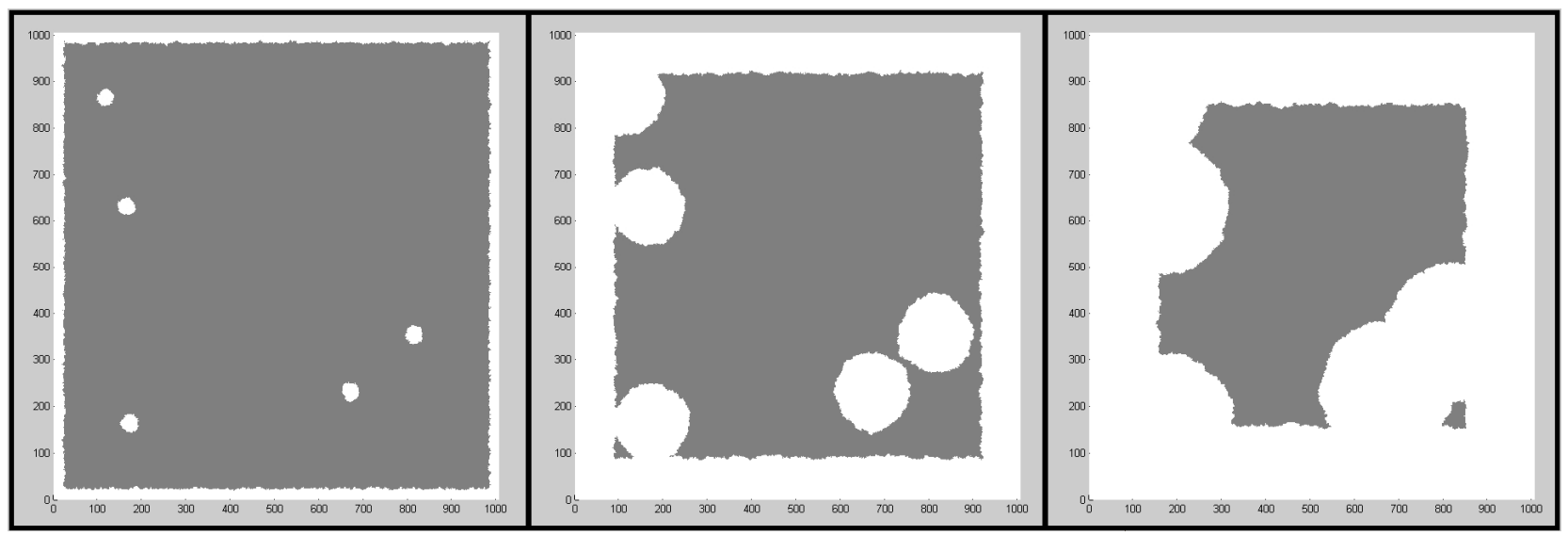

Figure 14: Reconstruction of flake structure with holes (SIM 2).

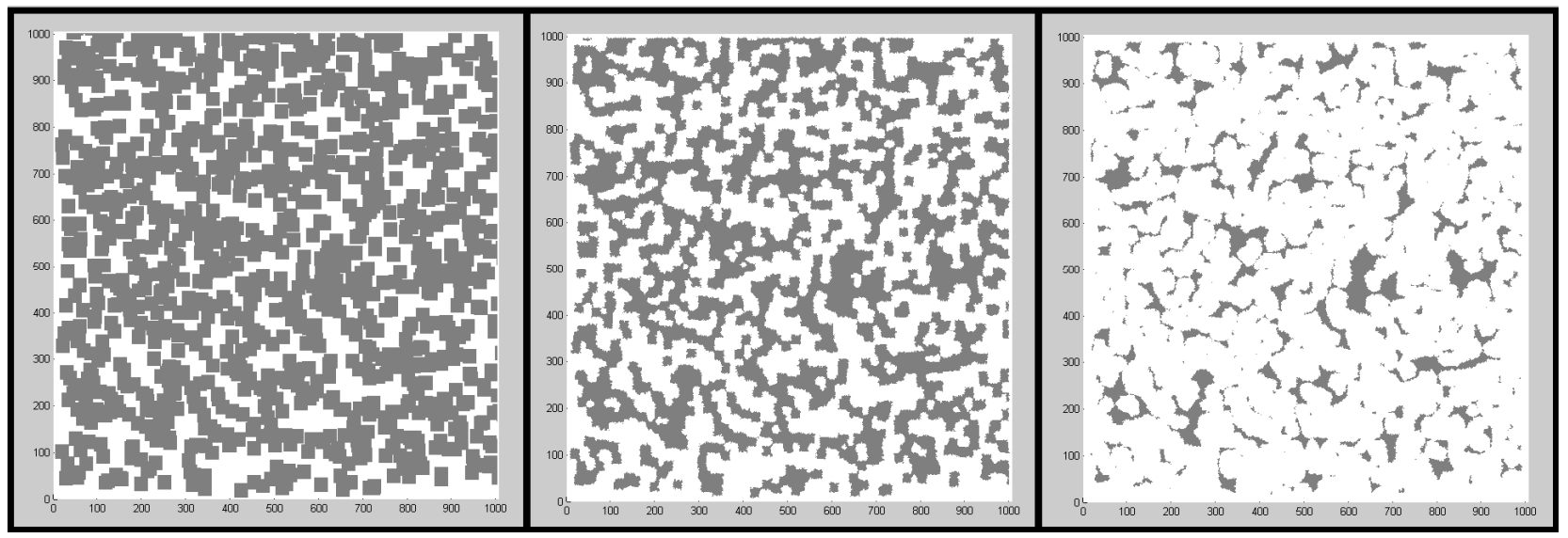

Figure 15: Reconstruction of flake structure with complex, erratic structure (SIM 3).

The fissures are simply represented by two slits, one sub-element wide, arranged in a cross on a square flake. For the second simulation small pits, one sub-element in diameter are placed within a square flake and allowed to grow. Since the holes are placed randomly in SIM 2, it is necessary to repeat the simulation to obtain an average. The average value for five repeat simulations is used to represent the ASA development of this particular reconstruction. Finally a fine erratic structure is created by building up a roughly square shaped particle from randomly placed smaller squares for SIM 3. Square flakes were chosen since it may be analytically shown that the conversion behaviour is identical to a circular disc.

\subsection{Simulation results}

The simulated conversion functions are divided by the surface roughening factor of 1.47 . The conversion functions for SIM 1 and 3 are shown in Figure 16. 


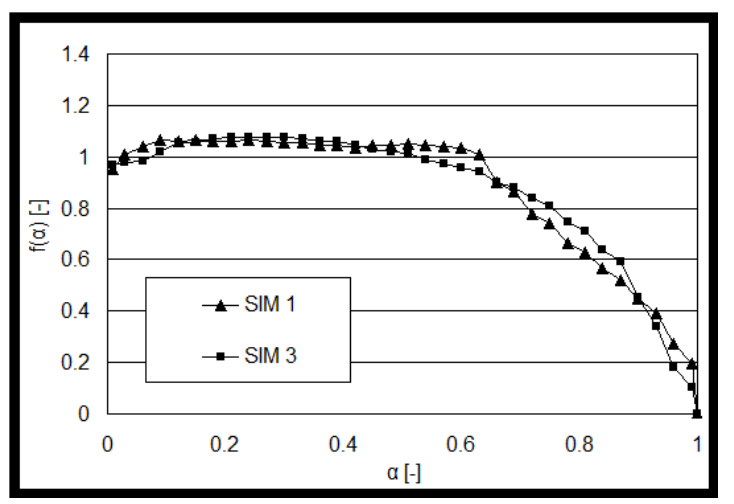

Figure 16: Simulated RFL conversion functions

These simulations are not capable of representing the initial increase of the RFL conversion function at low conversion. This region indicates a period of rapid ASA growth followed by a gradual decline. The RFL conversion function is compared to SIM 2 in Figure 17.

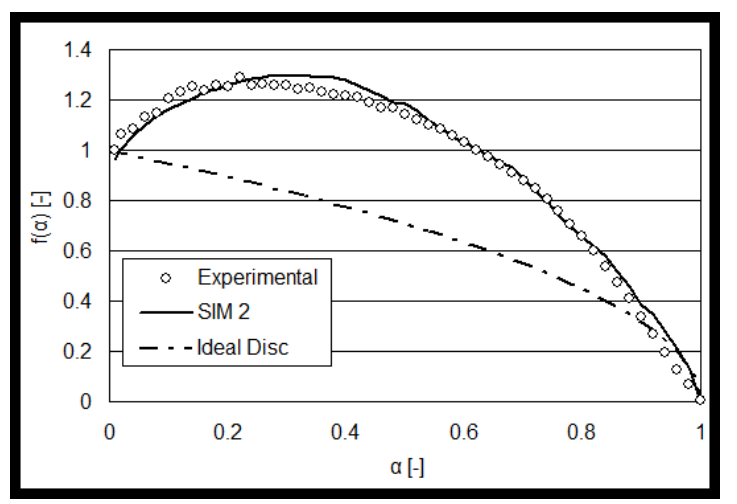

Figure 17: RFL conversion function and SIM 2

This model is the only version capable of accurately representing the observed conversion behaviour. The inability of SIM 1 to represent this behaviour is perhaps not unsurprising given the fact that the ASA growth afforded by the two fissures can at best counteract the shrinkage due to the four exposed edge sections. However, when additional fissures of similar length are added, the particle tends to rapidly fracture into segments, all of which are shrinking and hence no increase in the ASA is observed. On the other hand, the only difference between SIM 2 and SIM 3 is the defect density. For SIM 3 coalescence of the defects occurs too rapidly, similarly fracturing the particle into numerous tiny fragments, all of which are undergoing a decline in ASA.

In principle this demonstrates that the increase in ASA is due to the growth of a few discrete pits within the structure which creates sufficient additional area for reaction. As the pits coalesce the 
reaction rate subsequently declines to zero. This behaviour is reminiscent of the widely known random pore model [9]. For comparative purposes the analytical model of the ideal disc, which is monotonically decreasing, is also shown in this figure. It is clear that this model is not an adequate representation of the observed behaviour. However, if the disc model is subtracted from the experimental conversion function a behaviour similar to the conventional nucleation and growth model is observed [8]. When the pit formations shown in Figure 12B are examined more closely as in Figure 18, the catalytic particles responsible for the pitting can be seen.

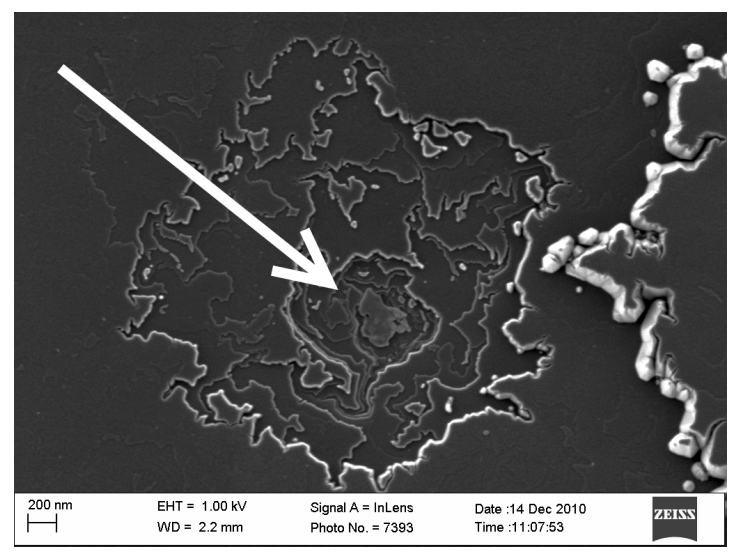

Figure 18: Pitting induced by catalytic impurities

Despite the high purity of the sample, it still contains particles which cause the pitting. Without the simulation it would have been difficult to determine whether this is a rate determining factor. Furthermore it may be possible to reduce the oxidation rate by removing these particles, potentially restoring the conversion behaviour to that of the ideal disc.

\section{Conclusions}

The active surface area progression which takes place in natural graphite flakes during oxidation is difficult to model analytically. A probability based simulation was developed to represent the observed behaviour. The simulation sub-divides a given geometry into a finite element type grid and is capable of easily representing very complex geometries which are difficult and time consuming to represent analytically. The probability used in the simulation was directly linked to the experimentally determined kinetic parameters. To validate the simulation it was shown to adequately describe the analytical model for a shrinking disc. Due to the discrete nature of the simulation it undergoes an initial surface roughening effect which has to be taken into consideration. This effect increases the reaction rate by a factor of 1.47 , which is fixed for a given reaction probability and grid size. 
A natural graphite sample was characterized using the ICTAC recommended approach for solid state reactions. The activation energy and pre-exponential factors were determined. These values and dimensions which are representative of the flakes were used in the simulation. The sample was partially oxidized and extensively examined under a scanning electron microscope. Three characteristic microstructures were identified in the oxidized sample. These structures were qualitatively approximated by three simulated structures. Two of the three simulations were unable to effectively represent the initial region of the conversion function where it increases towards a maximum.

This behaviour was accurately represented by the simulation of a square flake with a few randomly positioned pits growing in the flake structure. The simulation shows that the initial increase is due to the growth of the pits within the larger flake structure. This creates additional reaction interface for the oxidation. The rate increases until the pits coalesce and then declines to zero. This behaviour is very similar to that of the random pore model. Since the pits were found to be caused by catalytic impurities, this provides the practical insight that if the impurities can be removed, the oxidation resistance can be significantly improved. This may potentially transform the conversion behaviour into the monotonically decreasing behaviour expected from the ideal disc model.

This simulation was developed for the oxidation of graphite but can easily be applied to the gasification of any particulate material with a two dimensional structure. The model is simple and easy to use yet capable of representing complex geometries such as randomly curved contours. Some catalytically active impurities in graphite tend to trace randomly shaped channels across the graphite surface. Future work is focused on using the simulation to understand the impact of this behaviour, which would be very difficult to represent analytically. In addition, the matrix will be extended to a hexagonal shape more representative of the graphite crystal structure whilst developing an improved reaction probability density function based on the number of nearest neighbours.

\section{References}

[1] Badenhorst H, Rand B, Focke WW. A generalized solid state kinetic expression for reaction interface-controlled reactivity. Thermochim. Acta. 2013;562:1-10.

[2] Walker PL, Rusinko F, Austin LG. Gas Reactions of Carbon. Advances in Catalysis and Related subjects. 1959;11:133-221.

[3] Reynolds WN. Physical Properties of Graphite. Amsterdam: Elsevier; 1968. 
[4] Knight DS, White WB. Characterization of diamond films by Raman spectroscopy. J Mater Res 1989;4:385-93.

[5] Roscoe C. Catalytic oxidation of Ticonderoga graphite crystals. Carbon 1968;6:365-72.

[6] Wissler M. Graphite and carbon powders for electrochemical applications. Journal of Power Sources. 2006;156:142-50.

[7] Galwey AK, Brown ME. Thermal decomposition of ionic solids. Amsterdam: Elsevier; 1999.

[8] Vyazovkin S, Burnham AK, Criado JM, Pérez-Maqueda LA, Popescu C, Sbirrazzuoli N. ICTAC Kinetics Committee recommendations for performing kinetic computations on thermal analysis data. Thermochimica Acta. 2011;520(1-2):1-19.

[9] Bhatia SK, Perlmutter DD. A random pore model for fluid-solid reactions: I. isothermal, kinetic control. AIChE Journal. 1980;26:379-85. 